\title{
Carbon nanotube fiber terahertz polarizer
}

Ahmed Zubair, ${ }^{1}$ Dmitri E. Tsentalovich, ${ }^{2}$ Colin C. Young, ${ }^{2}$ Martin S. Heimbeck, ${ }^{3}$ Henry O. Everitt, 1,3 Matteo Pasquali, 2, 4,5 and Junichiro Kono ${ }^{1,5,6, a)}$

1) Department of Electrical and Computer Engineering, Rice University, Houston, Texas 7r005, USA

2) Department of Chemical and Biomolecular Engineering, Rice University, Houston, Texas 77005, USA

${ }^{3)}$ Charles M. Bowden Laboratory, Aviation \& Missile Research, Development, and Engineering Center (AMRDEC), Redstone Arsenal, Alabama 35898, $U S A$

4) Department of Chemistry, Rice University, Houston, TX r7005, $U S A$

5) Department of Materials Science and NanoEngineering, Rice University, Houston, Texas 77005, USA

6) Department of Physics and Astronomy, Rice University, Houston, Texas 7ro05, $U S A$

(Dated: 27 March 2016) 
Conventional, commercially available terahertz $(\mathrm{THz})$ polarizers are made of uniformly and precisely spaced metallic wires. They are fragile and expensive, with performance characteristics highly reliant on wire diameters and spacings. Here, we report a simple and highly error-tolerant method for fabricating a freestanding $\mathrm{THz}$ polarizer with nearly ideal performance, reliant on the intrinsically one-dimensional character of conduction electrons in well-aligned carbon nanotubes (CNTs). The polarizer was constructed on a mechanical frame over which we manually wound acid-doped CNT fibers with ultrahigh electrical conductivity. We demonstrated that the polarizer has an extinction ratio of $\sim-30 \mathrm{~dB}$ with a low insertion loss $(<0.5 \mathrm{~dB})$ throughout a frequency range of $0.2-1.1 \mathrm{THz}$. In addition, we used a $\mathrm{THz}$ ellipsometer to measure the Müller matrix of the CNT-fiber polarizer and found comparable attenuation to a commercial metallic wire-grid polarizer. Furthermore, based on the classical theory of light transmission through an array of metallic wires we demonstrated the most striking difference between the CNT-fiber and metallic wire-grid polarizers: the latter fails to work in the zero-spacing limit, where it acts as a simple mirror, while the former continues to work as an excellent polarizer even in that limit due to the one-dimensional conductivity of individual CNTs.

Keywords: terahertz, carbon nanotubes, carbon nanotube fiber, polarizers

a)kono@rice.edu; corresponding author. 
Unique properties of electromagnetic waves in the terahertz $(\mathrm{THz})$ frequency range are expected to lead to new applications in diverse areas, ${ }^{1-3}$ including wireless communications, chemical detection, and medical imaging. Recent advances in the generation, manipulation, and detection of $\mathrm{THz}$ waves have brought these anticipated applications closer to realization. However, $\mathrm{THz}$ technologies are still immature as compared to those available in different spectral ranges, and more sophisticated schemes and architectures as well as novel materials are being sought for improving operation performance and for making devices more compact and easier to fabricate.

Polarizers are essential components in THz imaging, communications, and spectroscopy. $\mathrm{THz}$ polarizers made of various materials have been reported, ${ }^{4-9}$ but most commercially available THz polarizers are metallic wire-grid polarizers, whose extinction ratios and insertion losses sensitively depend on the wire widths and spacings. Carbon nanotubes (CNTs) are emerging optoelectronic materials ${ }^{10}$ that have unique ultrabroadband anisotropic optical absorption properties. In particular, they exhibit essentially perfect polarization anisotropy in the THz frequency range, ${ }^{7-9,11}$ ideally suited for polarizer applications. Multifunctional architectures of highly aligned CNTs, such as fibers with ultrahigh conductivity ${ }^{12}$ and currentcarrying capacity ${ }^{13}$ synthesized by wet processing methods, are now available and promising as new polarizer wires.

Here, we describe a simple and robust method for fabricating a $\mathrm{THz}$ polarizer with nearly ideal performance, made of a hand-wound array of conductive fibers of well-aligned CNTs. The polarizer exhibited an extinction ratio $(E R)$ of $\sim-30 \mathrm{~dB}$ in a frequency range of $0.2-$ 1.1 THz. We measured spectra of Müller matrix elements for the polarizer using a frequencydomain $\mathrm{THz}$ ellipsometer, which showed that this polarizer has properties comparable to a commercial wire-grid polarizer. Being freestanding, this polarizer has a small insertion loss $(<0.5 \mathrm{~dB})$, as compared to previously reported $\mathrm{THz}$ polarizers based on thin films of aligned CNTs on substrates ${ }^{7,9}$ or on aligned multiwall CNT areogel sheets. ${ }^{8}$ In addition, the current method does not involve any complicated transfer and/or stacking processes that are time consuming and require careful alignment.

Figure 1 shows images of our THz polarizer. The polarizer was fabricated by manually winding a continuous, uniform, and ultralong $(>20 \mathrm{~m}) \mathrm{CNT}_{\text {fiber }}{ }^{12}$ with an average diameter of $12.6 \mu \mathrm{m}$ over a copper frame (Fig. 1a) to form two parallel layers with a suspended area of $5 \times 10 \mathrm{~mm}^{2}$ (Fig. 1b) and a layer spacing of $1 \mathrm{~mm}$. Each layer consisted of a dense array of 

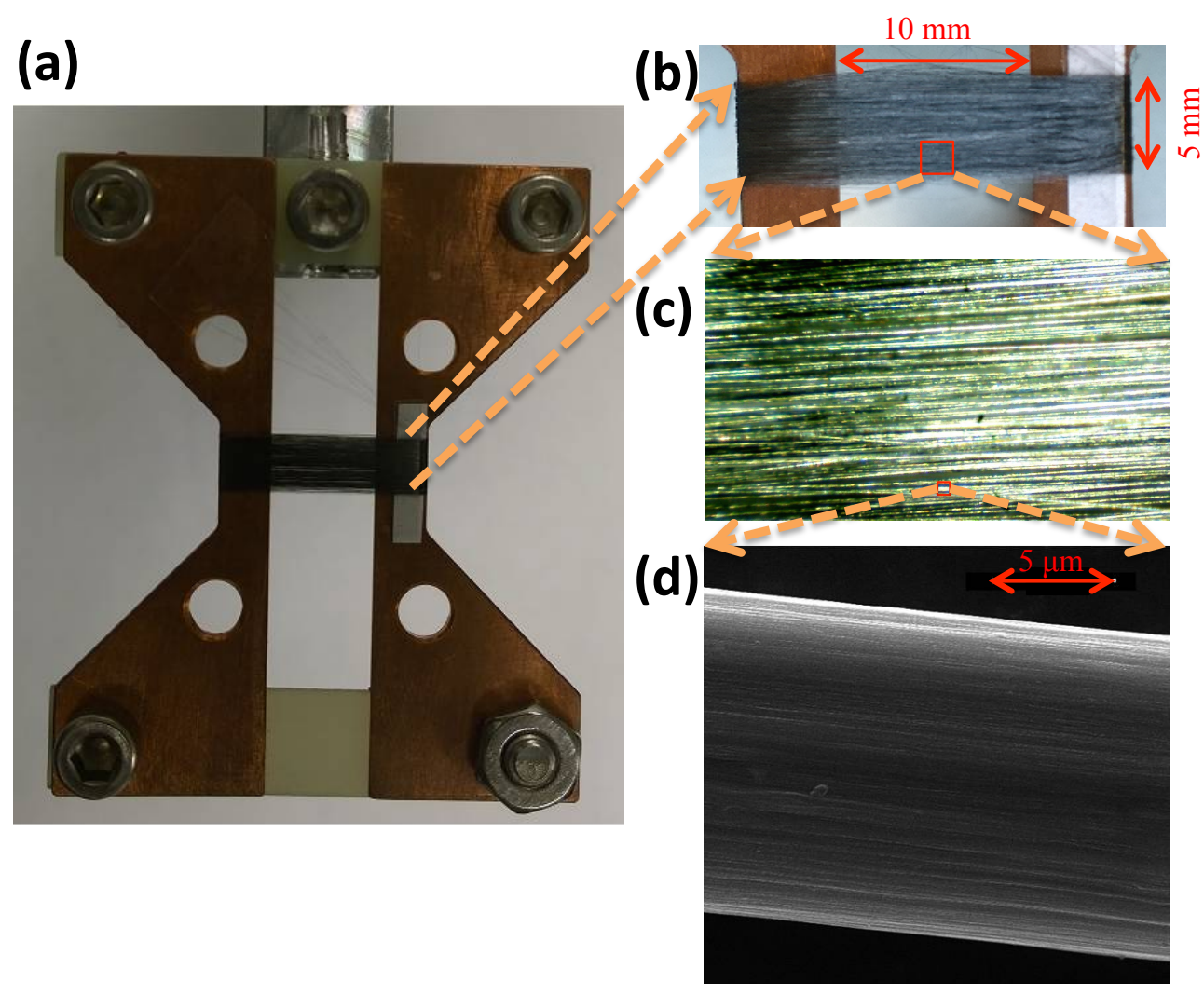

FIG. 1. Optical and scanning electron microscopy images of CNT-fiber THz polarizer. (a) Copper frame over which the fiber was wound. (b) The suspended area of the polarizer consisting of two parallel layers, each containing $\sim 400$ parallel CNT fibers. (c) Magnified view of the suspended area, showing densely packed fibers. (d) High magnification scanning electron microscopy image of a CNT fiber, showing excellent alignment of nanotubes.

400 parallel CNT fibers; i.e., each layer was designed to be a monolayer of parallel fibers with zero average spacing. Because the fiber was manually wound, it was impossible to keep the structure perfect (Fig. 1c); however, the structural imperfections did not negatively impact the performance of the fabricated polarizer, as detailed below. The CNT fibers had high electrical conductivity ( $~ 0.1$ that of copper) because they consist of highly aligned (Fig. 1d), densely-packed, low-defect acid-doped CNTs. ${ }^{12,14}$

We performed $\mathrm{THz}$ transmission measurements through the suspended area of the polarizer with a normal incident $\mathrm{THz}$ beam. ${ }^{14}$ Figure 2 a shows time-domain waveforms for $\mathrm{THz}$ pulses transmitted through the polarizer for parallel, perpendicular, and $45^{\circ}$ polarizations as well as for dry air (reference). For the perpendicular polarization, the transmitted $\mathrm{THz}$ 

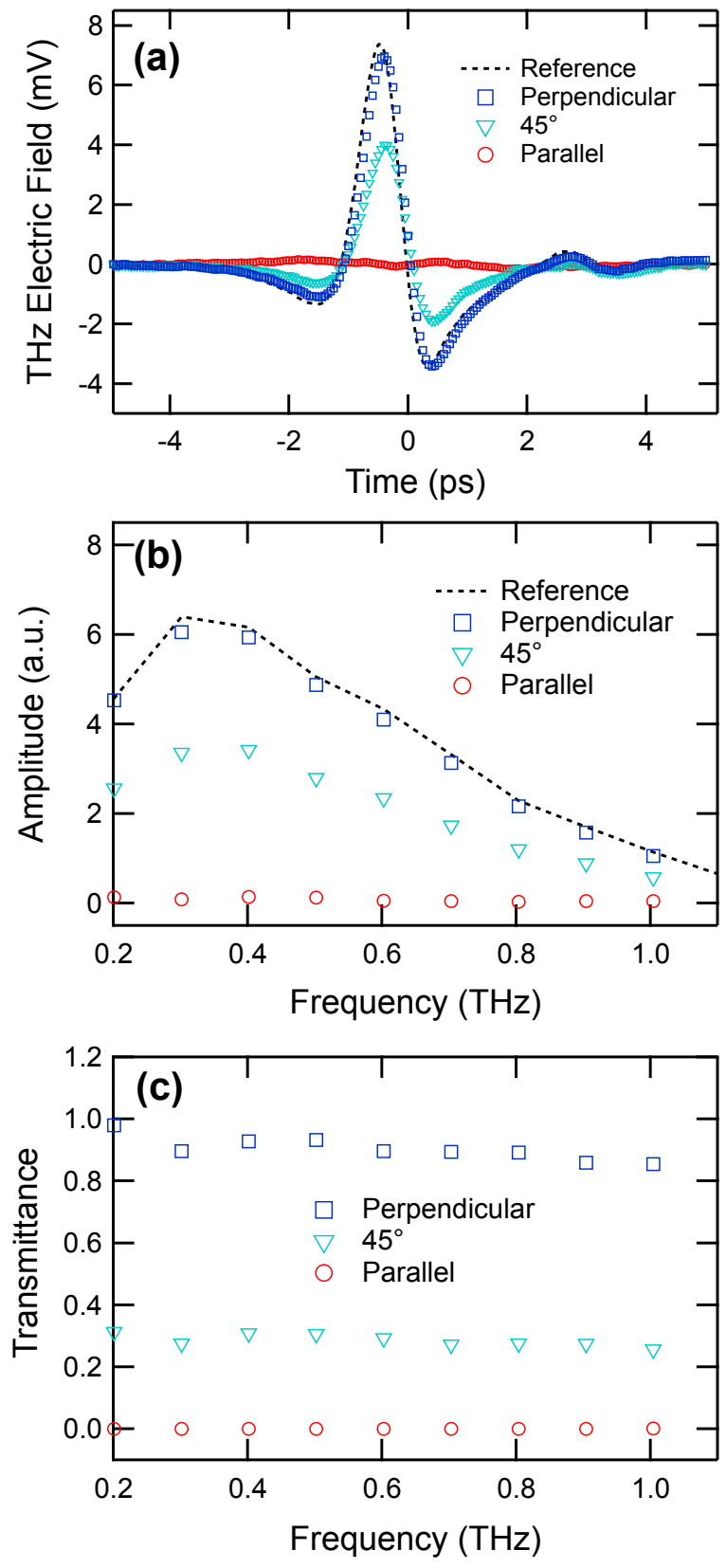

FIG. 2. (a) Time-domain waveforms for $\mathrm{THz}$ radiation transmitted through the CNT-fiber polarizer for parallel polarization (red circles), $45^{\circ}$ polarization (green triangles), and perpendicular polarization (blue squares), together with the waveform transmitted through dry air (black dashes). (b) Frequency-domain signal spectra for the dry-air reference (black dashes), parallel polarization (red circles), $45^{\circ}$ polarization (green triangles), and perpendicular polarization (blue squares). (c) Transmittance spectra for parallel polarization (red circles), $45^{\circ}$ polarization (green triangles), and perpendicular polarization (blue squares). 
signal is close to that for the reference, indicating that there is little attenuation. On the other hand, transmission is completely suppressed for the case of parallel polarization. The same trends can be seen in the frequency domain after Fourier transformation, as shown in the amplitude spectra (Fig. 2b) and transmittance spectra (Fig. 2c).

Figure 3a shows the attenuation spectra of the polarizer for parallel polarization (red circles) and perpendicular polarization (blue squares). The attenuation is defined as $A=$ $-\log _{10} T$, where $T$ is the transmittance. Figure $3 \mathrm{~b}$ shows the degree of polarization, $D O P=$ $\frac{T_{\|}-T_{\perp}}{T_{\|}+T_{\perp}}$, as well as the extinction ratio, $E R=\frac{T_{\|}}{T_{\perp}}$, where $T_{\|}\left(T_{\perp}\right)$ is the transmittance for the parallel (perpendicular) polarization. The DOP value is almost $100 \%$ throughout the entire frequency range. The $E R$ value is less than $10^{-3}$ (or $-30 \mathrm{~dB}$ ), with the minimum and average values $-37.1 \mathrm{~dB}$ and $-33.3 \mathrm{~dB}$, respectively. The average value for $T_{\perp}$ in this spectral range is $90 \%$ (Fig. 2c), so the average insertion loss $\left(-\log _{10} T_{\perp}\right)$ is $0.45 \mathrm{~dB}$, a value two or six times smaller than that reported by Ren et $a l^{9}{ }^{9}$ or Kyoung et $a l .^{8}$, respectively.

For a more complete characterization of the polarizer, we further performed continuouswave transmission measurements using a solid-state frequency-domain $\mathrm{THz}$ source and super-heterodyne detector system based on the frequency multiplication of a $13-20 \mathrm{GHz}$ microwave local oscillator. ${ }^{15}$ Polarimetric transmission was measured in a frequency range of $0.2-0.8 \mathrm{THz}$, and the $E R$ was computed. As can be seen in Fig. 3c, this experiment also showed $E R$ values of $\sim-30 \mathrm{~dB}$ for the CNT fiber polarizer (red crosses). Also shown in Fig. 3c are data taken for a reference commercial free-standing metallic wire-grid polarizer (black solid circles). The expected $-35 \mathrm{~dB} E R$ of the reference polarizer was obtained at $0.8 \mathrm{THz}$, thereby validating the calibration of the ellipsometer and demonstrating that the CNT polarizer has comparable performance to the commercial polarizer.

Furthermore, we used a third $\mathrm{THz}$ measurement system, a frequency-domain $\mathrm{THz}$ ellipsometer, ${ }^{16}$ which allowed us to measure the Müller matrix ${ }^{17}$ of the CNT-fiber polarizer. The Müller matrix of an optical sample is a real-valued $4 \times 4$ matrix that completely describes the polarization properties of a material by the way it transforms the four-element polarimetric Stokes vector of the incident beam to the Stokes vector after its interaction 

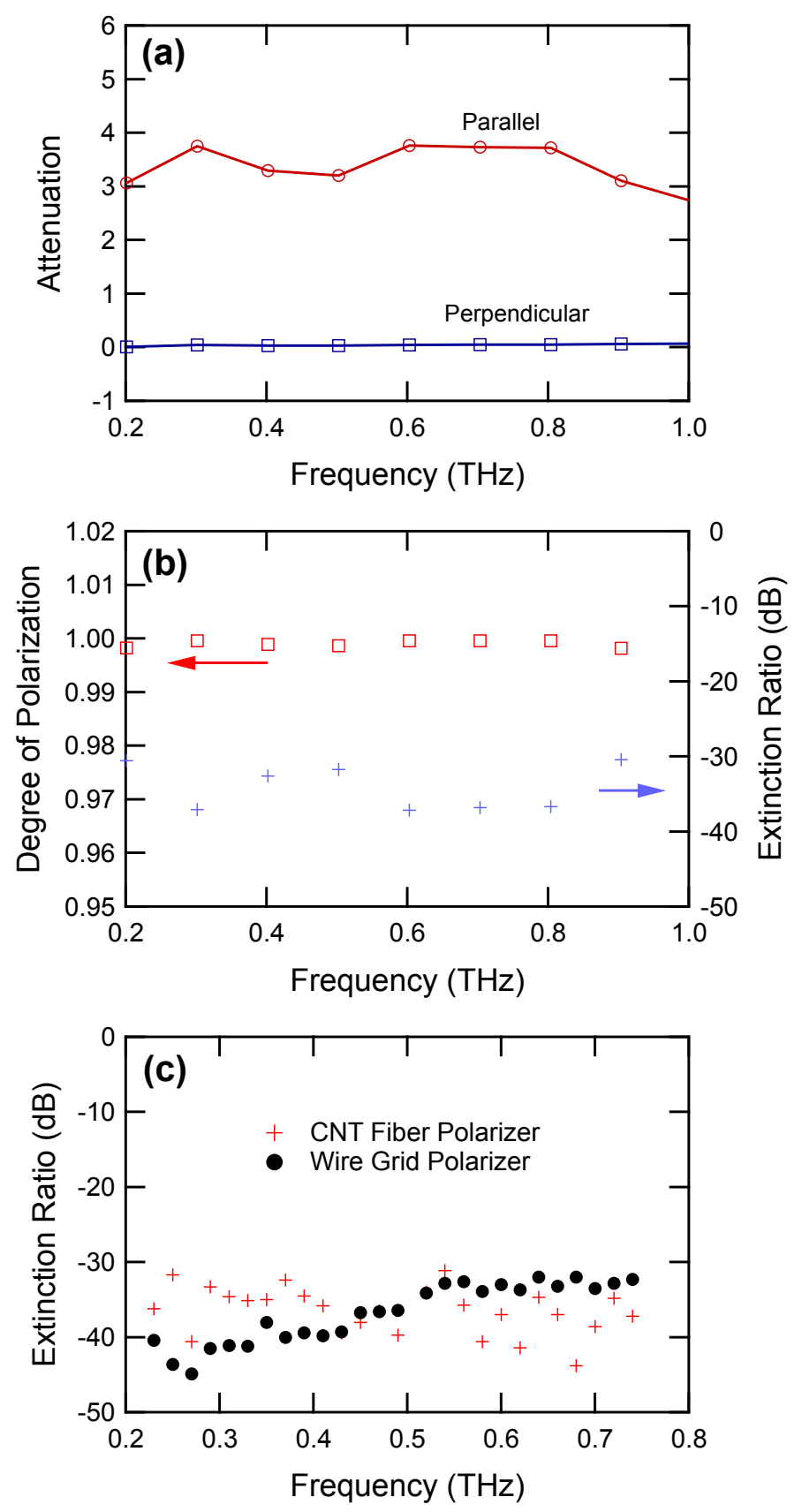

FIG. 3. (a) Attenuation spectra of the CNT-fiber polarizer for parallel polarization (red circles) and for perpendicular polarization (blue squares) in a frequency range of 0.2-1.1 THz. (b) Degree of polarization and extinction ratio of the polarizer in the $0.2-1.1 \mathrm{THz}$ range. The degree of polarization is basically $100 \%$, and the average extinction ratio in this frequency range is $-33.5 \mathrm{~dB}$. (c) Extinction ratio of the CNT-fiber polarizer (red crosses) and a commercial metallic wire-grid polarizer (black solid circles) in in a frequency range of $0.2-0.8 \mathrm{THz}$, taken with a frequency-domain $\mathrm{THz}$ ellipsometer. 

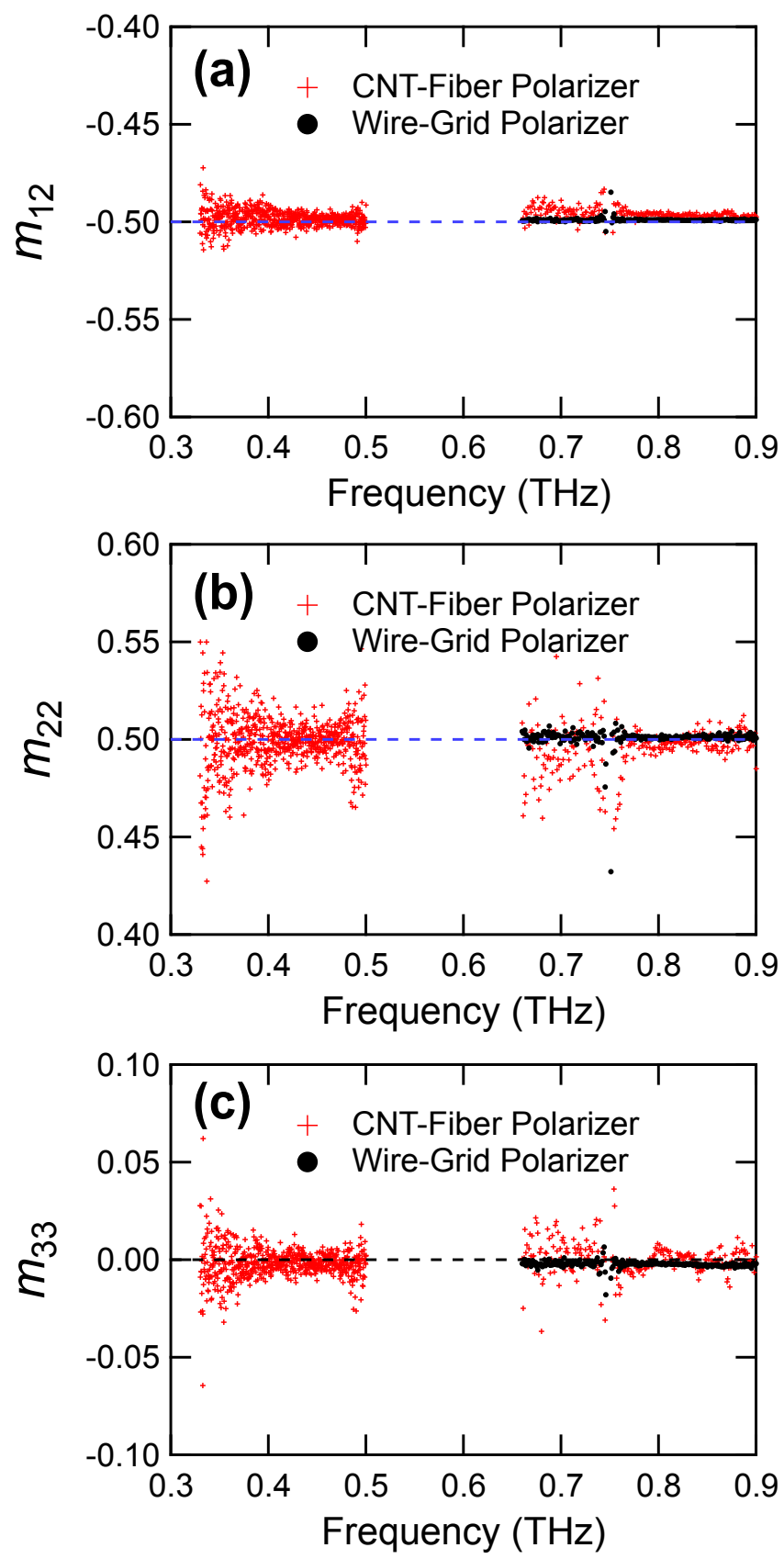

FIG. 4. Significant Müller matrix elements, (a) $m_{12}$, (b) $m_{22}$, and (c) $m_{33}$, measured for the CNTfiber polarizer (red crosses) and a commercial metallic wire-grid polarizer (black solid circles) as a function of frequency using a frequency-domain $\mathrm{THz}$ ellipsometer. 
with the material. ${ }^{14}$ Figure 4 shows the significant elements of the Müller matrix given by ${ }^{17}$

$$
\begin{aligned}
& m_{12}=\frac{T_{\|}^{2}-T_{\perp}^{2}}{2} \\
& m_{22}=\frac{T_{\|}^{2}+T_{\perp}^{2}}{2} \\
& m_{33}=T_{\|} T_{\perp}
\end{aligned}
$$

where $m_{i j}$ is the $i j$-th element of the Müller matrix, $i(j)$ being the row (column) number. For an ideal polarizer, $T_{\|}=0$ and $T_{\perp}=1$, and therefore, $m_{12}=-0.5, m_{22}=0.5$, and $m_{33}$ $=0$. As shown in Fig. 4, the CNT polarizer exhibits these ideal values.

Finally, we emphasize that the CNT fibers in our polarizer are disordered with irregularities and that the average spacing between adjacent fibers is zero. These facts are striking in light of the nearly ideal polarizer characteristics we demonstrated above. In the case of a metallic wire-grid polarizer, such irregularities and zero spacing would be detrimental to its performance. To further demonstrate this point (particularly, the zero-spacing limit), we performed simulations of transmission of polarized light through an array of metallic wires with varying spacings. ${ }^{14}$ Figure 5 a shows the calculated transmittance values, $T_{\|}$and $T_{\perp}$, at $0.5,1$, and $2 \mathrm{THz}$ as a function of spacing. At all frequencies, the value of $T_{\perp}$ rapidly decreases to zero as the spacing approaches zero, while $T_{\|} \approx 0$ for all spacings. The corresponding insertion loss, $-\log _{10} T_{\perp}$, is plotted in Fig. 5b, which increases with decreasing spacing and becomes infinite in the zero spacing limit. These results highlight the uniqueness of the CNT-fiber polarizer, as compared to conventional wire-grid polarizers: the latter's working principle is based on the structural anisotropy of wires, whereas the former is reliant on the intrinsically one-dimensional character of conduction electrons in CNTs. For $\mathrm{THz}$ and infrared measurements of optical conductivity of CNTs, see, e.g., Ref. ${ }^{18}$.

In conclusion, we developed a very simple method for fabricating an ideal $\mathrm{THz}$ polarizer based on fibers of highly-conductive and well-aligned carbon nanotubes. Its performance was fully characterized through measurement of salient Müller matrix elements and shown to be comparable to commercial metallic wire-grid polarizers. Its performance characteristics are tolerant to structure imperfections introduced in the hand-winding process, making this a robust method for producing high-quality THz polarizers in a cost-effective manner. The intrinsic anisotropic optical properties of aligned CNTs are the reason for this ideal performance. This CNT-fiber polarizer will have important impact in emerging $\mathrm{THz}$ tech- 

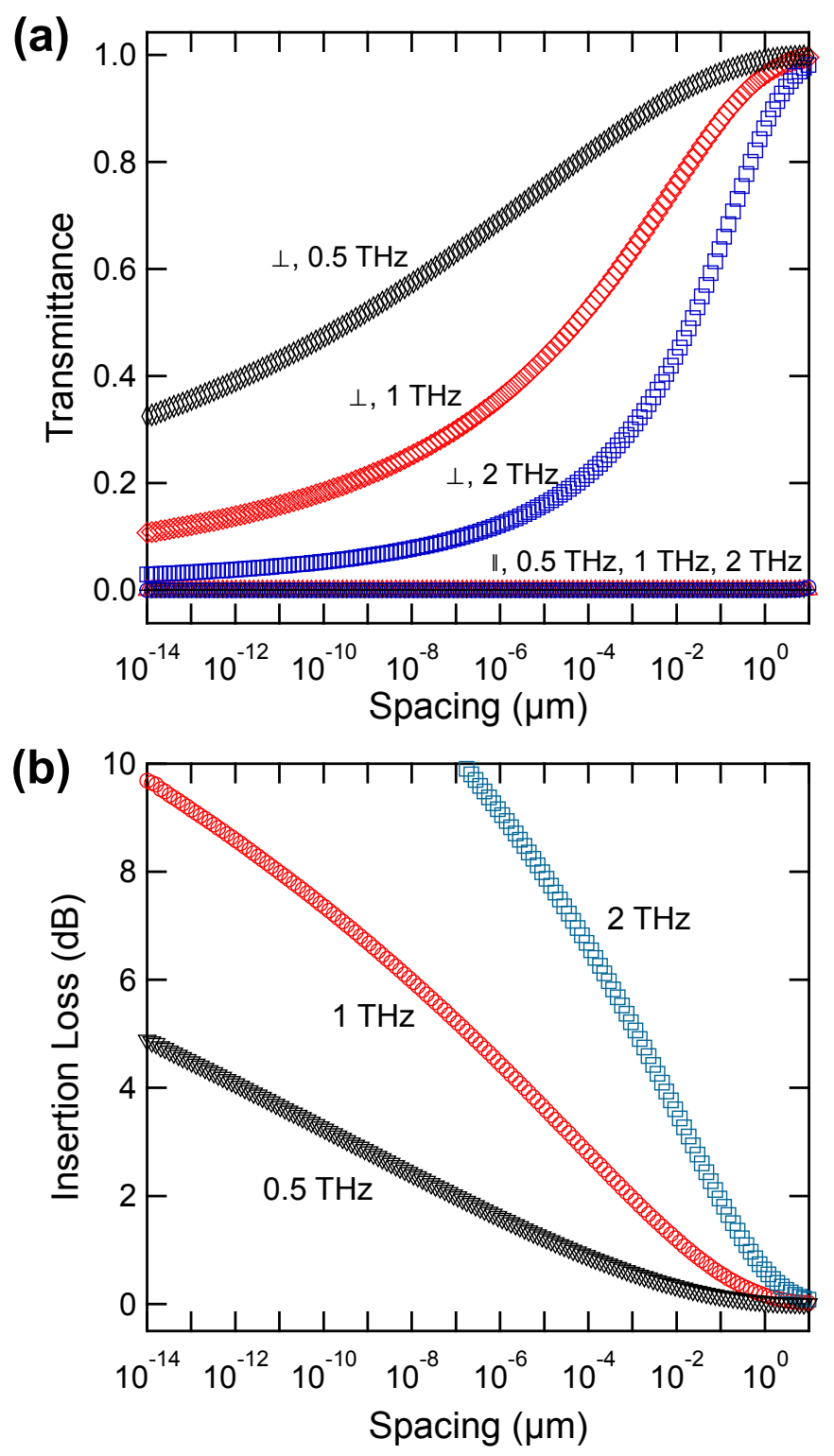

FIG. 5. (a) Calculated transmittance for polarized light with frequencies of $0.5,1$, and $2 \mathrm{THz}$ through a periodic array of 12.6 - $\mu \mathrm{m}$-diameter metallic wires with infinite conductivity as a function of wire spacing. (b) Calculated insertion loss (in $\mathrm{dB}$ ) as a function of wire spacing. In the limit where adjacent wires touch each other, the insertion loss becomes infinitely high, turning the polarizer into a mirror.

nological applications such as wireless communications, imaging, chemical detection, and astrophysics. 


\section{ACKNOWLEDGMENTS}

We thank W. Caraway for his help with the ellipsometer measurements. A.Z. and J.K. were supported by DOE BES through Grant No. DE-FG02-06ER46308 (THz characterization of CNT fibers), the Robert A. Welch Foundation Grant No. C-1509 (sample preparation), and the W. M. Keck Foundation. M.S.H. and H.O.E. were supported by the Army Inhouse Laboratory Independent Research Program. D.E.T., C.C.Y., and M.P. were supported by Teijin Aramids B.V., Teijin Techno Products, and AFOSR through Grant No. FA9550-091-0590. Conflict of Interest: We declare the following competing financial interest(s): M.P., D.E.T., and C.C.Y. are authors of the PCT international application PCT/EP2012/067478 describing the method for manufacturing CNT fibers by wet spinning. M.P. and D.E.T. have a financial interest in DexMat, Inc., which is commercializing wet-spun CNT fibers.

\section{REFERENCES}

${ }^{1}$ K. Sakai, ed., Terahertz Optoelectronics (Springer, Berlin, 2005).

${ }^{2}$ D. M. Mittleman, ed., Sensing with Terahertz Radiation (Springer, Berlin, 2003).

${ }^{3}$ P. H. Siegel, IEEE Trans. Microwave Theor. Tech. 50, 910 (2002).

${ }^{4}$ E. Costley, K. H. Hursey, G. F. Neill, and J. M. Ward, J. Opt. Soc. Am. 67, 979 (1977).

${ }^{5}$ A. Wojdyla and G. Gallot, Opt. Exp. 19, 14099 (2011).

${ }^{6}$ C.-F. Hsieh, Y.-C. Lai, R.-P. Pan, and C.-L. Pan, Opt. Lett. 33, 1174 (2008).

${ }^{7}$ L. Ren, C. L. Pint, L. G. Booshehri, W. D. Rice, X. Wang, D. J. Hilton, K. Takeya, I. Kawayama, M. Tonouchi, R. H. Hauge, and J. Kono, Nano Lett. 9, 2610 (2009).

${ }^{8}$ J. Kyoung, E.-Y. Jang, M. D. Lima, H.-R. Park, R.-O. Robls, X. Lepro, Y.-H. Kim, R. H. Baughman, and D.-S. Kim, Nano Lett. 11, 4227 (2011).

${ }^{9}$ L. Ren, C. L. Pint, T. Arikawa, K. Takeya, I. Kawayama, M. Tonouchi, R. H. Hauge, and J. Kono, Nano Lett. 12, 787 (2012).

${ }^{10}$ S. Nanot, E. H. Hároz, J.-H. Kim, R. H. Hauge, and J. Kono, Adv. Mater. 24, 4977 (2012).

${ }^{11}$ L. Ren, Q. Zhang, C. L. Pint, A. K. Wójcik, M. Bunney, T. Arikawa, I. Kawayama, M. Tonouchi, R. H. Hauge, A. A. Belyanin, and J. Kono, Phys. Rev. B 87, 161401(R) (2013). 
${ }^{12}$ N. Behabtu, C. C. Young, D. E. Tsentalovich, O. Kleinerman, X. Wang, A. W. K. Ma, E. A. Bengio, R. F. ter Waarbeek, J. J. de Jong, R. E. Hoogerwerf, S. B. Fairchild, J. B. Ferguson, B. Maruyama, J. Kono, Y. Talmon, Y. Cohen, M. J. Otto, and M. Pasquali, Science 339, 182 (2013).

${ }^{13}$ X. Wang, N. Behabtu, C. C. Young, D. E. Tsentalovich, M. Pasquali, and J. Kono, Adv. Func. Mater. 24, 3241 (2014).

${ }^{14}$ See supplemental material at [URL will be inserted by AIP] for more detailed information on the methods used.

${ }^{15}$ T. W. Crowe, D. W. Porterfield, J. L. Hesler, W. L. Bishop, D. S. Kurtz, and K. Hui, Proc. SPIE 5790, 271 (2005).

${ }^{16}$ T. Hofmann, C. M. Herzinger, A. Boosalis, T. E. Tiwald, J. A. Woollam, and M. Schubert, Rev. Sci. Instrum. 81, 023101 (2010).

${ }^{17}$ D. Goldstein, Polarized Light, 2nd ed. (Marcel Dekker, Inc., New York, 2003).

${ }^{18}$ R. R. Hartmann, J. Kono, and M. E. Portnoi, Nanotechnology 25, 322001 (2014). 